\title{
A!
}

This is an electronic reprint of the original article.

This reprint may differ from the original in pagination and typographic detail.

Rossi, Tuomas P.; Zugarramurdi, Asier; Puska, Martti J.; Nieminen, Risto M.

\section{Quantized Evolution of the Plasmonic Response in a Stretched Nanorod}

Published in:

Physical Review Letters

DOI:

10.1103/PhysRevLett.115.236804

Published: 01/12/2015

Document Version

Publisher's PDF, also known as Version of record

Please cite the original version:

Rossi, T. P., Zugarramurdi, A., Puska, M. J., \& Nieminen, R. M. (2015). Quantized Evolution of the Plasmonic Response in a Stretched Nanorod. Physical Review Letters, 115(23), 1-6. [236804].

https://doi.org/10.1103/PhysRevLett.115.236804

This material is protected by copyright and other intellectual property rights, and duplication or sale of all or part of any of the repository collections is not permitted, except that material may be duplicated by you for your research use or educational purposes in electronic or print form. You must obtain permission for any other use. Electronic or print copies may not be offered, whether for sale or otherwise to anyone who is not an authorised user. 


\title{
Quantized Evolution of the Plasmonic Response in a Stretched Nanorod
}

\author{
Tuomas P. Rossi, ${ }^{1,}{ }^{*}$ Asier Zugarramurdi, ${ }^{1}$ Martti J. Puska, ${ }^{1}$ and Risto M. Nieminen ${ }^{1,2}$ \\ ${ }^{1}$ COMP Centre of Excellence, Department of Applied Physics, Aalto University School of Science, \\ P.O. Box 11100, FI-00076 Aalto, Finland \\ ${ }^{2}$ Dean's Office, Aalto University School of Science, P.O. Box 11000, FI-00076 Aalto, Finland
}

(Received 2 June 2015; published 1 December 2015)

\begin{abstract}
Quantum aspects, such as electron tunneling between closely separated metallic nanoparticles, are crucial for understanding the plasmonic response of nanoscale systems. We explore quantum effects on the response of the conductively coupled metallic nanoparticle dimer. This is realized by stretching a nanorod, which leads to the formation of a narrowing atomic contact between the two nanorod ends. Based on firstprinciples time-dependent density-functional-theory calculations, we find a discontinuous evolution of the plasmonic response as the nanorod is stretched. This is especially pronounced for the intensity of the main charge-transfer plasmon mode. We show the correlation between the observed discontinuities and the discrete nature of the conduction channels supported by the formed atomic-sized junction.
\end{abstract}

PACS numbers: 73.20.Mf, 36.40.Gk, 73.23.-b, 78.67.-n

Metallic nanoparticles are of great interest because they have potential in many applications, such as biosensor development [1], spectroscopy [2], and nanobiomedicine [3]. This potential is largely due to their versatile optical properties, which are mainly determined by the localized surface plasmon resonances supported by the nanoparticle. These excitations are characterized by the collective oscillations of the delocalized electrons in response to light. Understanding the physics determining their dependence on nanoparticle parameters is thus a major subject of research, which seeks different routes to tailor materials with desired optical properties. Along with the size, shape, environment, and atomic composition of the individual nanoparticles, the plasmonic response can be modified by forming extended systems, such as nanoparticle arrays and agglomerates [4]. Among these, nanoparticle dimers are not only the simplest configurations for studying the plasmonic interparticle interaction; they also serve as basic components in the design of nanoantennas $[5,6]$.

The plasmonic response of large nanoparticle dimers can be mostly understood by classical electrodynamics and the plasmon hybridization models $[7,8]$. However, the classical description fails at subnanometer interparticle separations, where quantum tunneling of electrons strongly affects the plasmonic response. Recently, this quantum regime has become experimentally accessible $[9,10]$, confirming several theoretical predictions [11-13]. Follow-up studies have further addressed quantum effects from dimers of mesoscopic sizes [14] down to the atomistic details [15], and have also considered the formation of conductive contacts $[16,17]$ as well as molecular and atomic junctions between the nanoparticles [18-21].

In this Letter we report, based on first-principles calculations, that the plasmonic response of nanoparticle dimers shows important quantum features within the metal-contact regime. We consider a metallic nanorod subject to a stretching process which leads to the formation of a narrowing atomic contact between the two nanorod ends, until eventual breakage. Remarkably, the calculations reveal a discontinuous evolution of the plasmon energies and intensities during stretching. We find a strong correlation between the observed discontinuities and the quantized nature of the conductance of the narrow contact. This effect is pronounced for the main charge-transfer plasmon mode.

For our study, we consider a flat-edged sodium nanorod obtained by extracting 25 atomic layers from the $b c c$ lattice along the (001) direction. The resulting system, shown in panel $A$ of Fig. 1(a), comprises 261 atoms. The lattice constant was optimized to $4.14 \AA$ by energy minimization of the nanorod (see below for the numerical details), leading to an approximate diameter of $13 \AA$ and an aspect ratio of 4 . This size is close to the smallest metallic nanorods produced in recent experiments [22]. The use of sodium for modeling is not only computationally convenient because of the single valence electron per atom needed in its description; it has also been successful in reproducing many general phenomena of simple and noble metals in cluster science and plasmonics, within both atomistic [15-17,23,24] and continuum approaches $[11,13,25,26]$.

The initial perfect nanorod is adiabatically stretched by pulling apart the frozen ends in steps of $0.05 \AA$, as schematically indicated in panel $A$ of Fig. 1(a). After each elongation step, the atomic positions of the sandwiched central slab of 51 atoms are allowed to relax. The geometry relaxation is performed with density-functional theory [33] calculations using the GPAW code [34,35]. We use the Perdew-Burke-Ernzerhof (PBE) [36] exchange-correlation (xc) functional. Selected snapshots of the simulated 

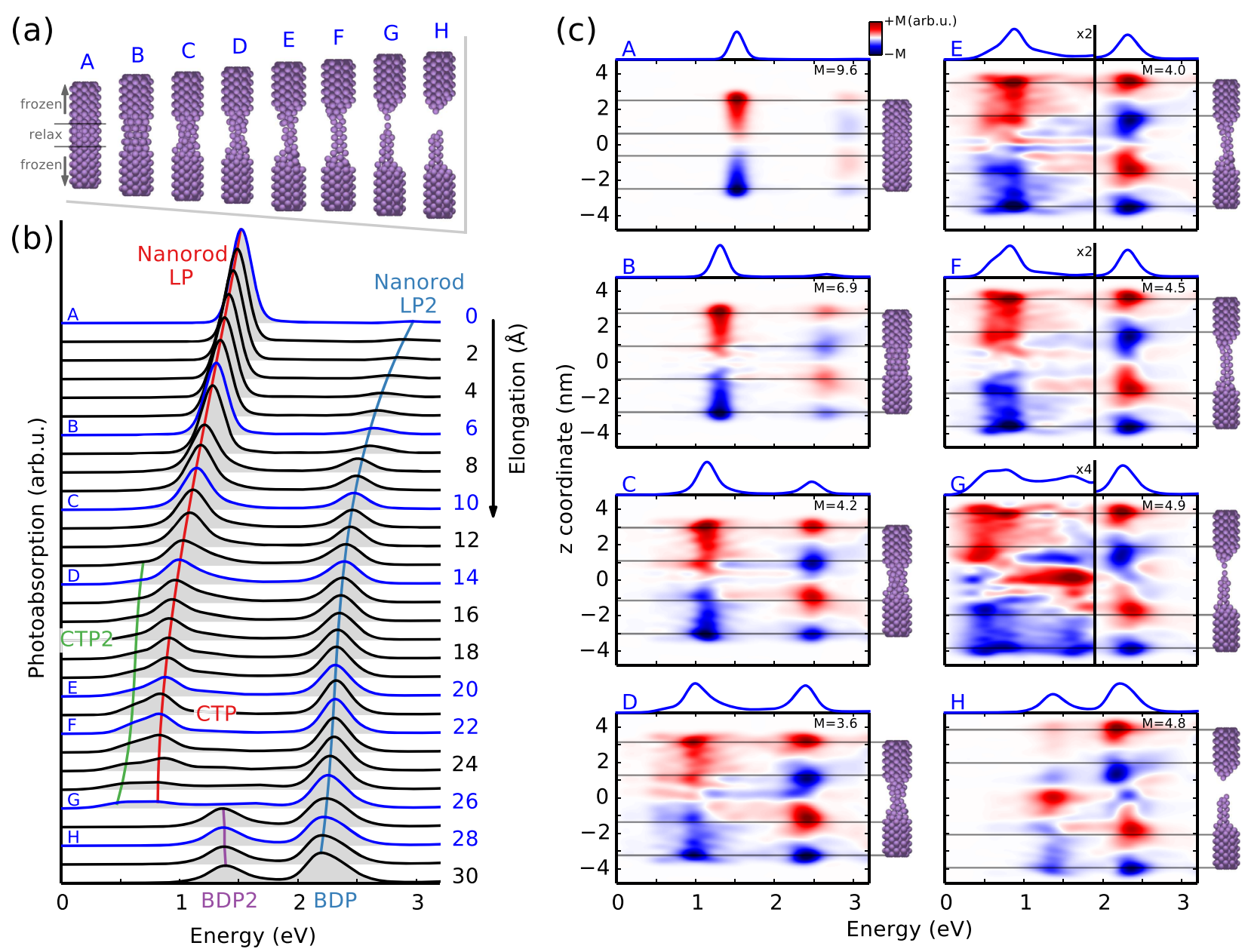

FIG. 1 (color online). Evolution of the plasmonic response of a sodium nanorod under stretching. (a) Selected snapshots of the simulated stretching process of the nanorod. Elongation distances $d$ from $A$ to $H$ : 0, 6, 10, 14, 20, 22, 26, and 28 A. (b) Longitudinal photoabsorption spectra of the stretched nanorod as a function of energy $\hbar \omega$. Solid lines following the plasmon modes (see main text for the label definitions) are drawn to guide the eye. (c) IID maps [see Eq. (2)] as a function of energy and $z$ coordinate along the main axis of the nanorod. The maps have been smoothed along $z$ to emphasize the gross features of the induced density [27]. Insets show the corresponding geometries (right) and spectra (top). Note that in panels $E, F$, and $G$, the low-energy part of the IID map and spectrum have been scaled by the indicated factor.

stretching process are shown in Fig. 1(a), labeled from $A$ to $H$. During the stretching, the break junction undergoes alternating sequences of elastic deformations and stochastic rearrangements. In this process, well-ordered structures can be formed in the junction, associated to nanowires with stable magic radii [25,37], a clear example of which is observed in panel $F$. Our simulation is in line with previous approaches studying the breaking of metallic nanowires $[24,25,38-40]$.

The optical properties of the described stretched nanorod are studied with time-dependent density-functional theory (TDDFT) [41] calculations. In brief, the time-dependent Kohn-Sham equations are solved in real time [42] with the adiabatic PBE xc functional as implemented in GPAW [43-45]. In this framework, the plasmons arise from the correlated time evolutions of the Kohn-Sham electrons in the mean field produced by other electrons. We consider only the longitudinal optical response along the main axis (z) of the nanorod. The initial ground-state electronic structure of the system is perturbed by a weak delta-pulse electric field. The subsequent computation of the time evolution of the valence electrons yields the induced charge density $\delta \rho(\boldsymbol{r}, t)$, from which the dynamic polarizability $\alpha(\omega)$ can be derived. The response is characterized via the photoabsorption cross section of the system

$$
\sigma_{\mathrm{abs}}(\omega)=\frac{\omega}{c \epsilon_{0}} \operatorname{Im}[\alpha(\omega)],
$$

where $c$ is the speed of light and $\epsilon_{0}$ the vacuum permittivity. The plasmon resonances appearing in the photoabsorption spectrum can be further analyzed by the imaginary part of the integrated induced density (IID) defined as 


$$
\delta \tilde{\rho}(z, \omega)=\operatorname{Im}\left[\int \delta \rho(\boldsymbol{r}, \omega) d x d y\right] .
$$

Note that the polarizability can be obtained from the IID via $\operatorname{Im}[\alpha(\omega)] \propto \int z \delta \tilde{\rho}(z, \omega) d z$. Thus, the IID serves as a $z$-resolved spectrum summarizing the spatial distribution of the plasmon modes.

We start our discussion by characterizing the different plasmon modes found in the calculated spectra shown in Fig. 1(b) for the increasing elongation distance $d$. The breaking of the narrowing junction after $d=26 \AA$ divides the spectra into two different regimes. We first focus on the contact regime $(d \leq 26 \AA)$, characterized by the progressive quenching and emergence of several different plasmon modes.

Initially $(d=0)$ the spectrum of the nanorod is governed by a longitudinal plasmon (LP) mode at $1.5 \mathrm{eV}$. The dipolar character of this mode can be recognized in its corresponding IID in panel $A$ of Fig. 1(c). Additionally, we observe a faint higher-order multipolar mode at $2.9 \mathrm{eV}$ (LP2). As the nanorod is stretched into a connected subnanorod dimer system, the evolved LP mode is better described as a charge-transfer plasmon (CTP), consistent with the literature on nanoparticle dimers [9-21,46-48]. The former label, LP, emphasizes the single nanorod character of the mode. On the other hand, the latter label, CTP, reflects that the mode can only be supported by the charge flow through the forming conductive junction between the nanorod ends. Additionally, the LP2 mode of the single nanorod evolves into a bonding dipolar plasmon (BDP) in the dimer, both modes having a similar nodal structure in the IID. The BDP mode arises from the hybridization $[8,46]$ of the dipolar plasmon modes of the two subnanorods. In contrast to the CTP, the BDP mode exists via capacitive coupling, also when the junction is removed. For increasing $d$, the LP-CTP mode quenches accompanied by a broadening, whereas the initially faint LP2-BDP mode grows, eventually becoming the dominant resonance in the response. Both peaks undergo strong redshifts, consistent with experiments and classical calculations performed on conductively connected metallic nanoparticles [48-51]. Thus, the redshifts can be ascribed to the combined effect of the simultaneous reduction of the diameter of the junction and the increasing length of the system.

For $d \gtrsim 13 \AA$, a second charge-transfer plasmon (CTP2) mode emerges at $\sim 0.3 \mathrm{eV}$ below the partially overlapping CTP peak. The CTP2 progressively grows with increasing $d$ and eventually becomes comparable in strength to the CTP mode. A careful inspection of the IID maps in Fig. 1(c) (panels $D-G$ ) reveals subtle differences between these two charge-transfer modes. While the CTP mode retains much of its dipolar character with a mostly localized IID in the far ends of the entire system, the CTP2 has a strong contribution from both the far and inner ends of the forming subnanorods. To further investigate these two modes, we performed additional calculations with different system sizes and junction geometries (not shown) suggesting that (i) the CTP2-like charge oscillation is likely to be dominant for thin junctions and (ii) the two modes possibly merge in larger systems, in agreement with classical calculations where a single dominant chargetransfer mode is observed $[49,50]$.

Right before breaking, at $d=26 \AA$, the stretched nanorod reaches the interesting limiting case of a monoatomic contact. In this configuration, the junction is almost detached from the upper subnanorod [see panel $G$ in Fig. 1(a)] and the CTP mode is strongly suppressed. In addition to the discussed charge-transfer plasmon modes, a broad structure of comparable strength is observed at $1.6 \mathrm{eV}$. The corresponding IID map $(G)$ in Fig. 1(c) reveals the contribution of the junction to the mode. Thus, this atomic configuration can be viewed as a heterogeneous trimer.

After the breaking of the junction $(d>26 \AA)$, the spectrum changes abruptly. We observe that the CTP modes disappear completely. This is in contrast with the progressive onset of the CTP modes supported by quantum tunneling when two metallic nanoparticles are brought into contact $[9,10]$. In our case, the quantum tunneling is rapidly suppressed after the breaking because of (i) the contraction of the broken junction towards the subnanorods, thus increasing the vacuum gap, and (ii) the small available tunneling area in the resulting geometry. Another major change in the spectrum is the appearance of a second bonding dipolar mode (BDP2) at $1.3 \mathrm{eV}$. From the IID map shown in panel $H$ of Fig. 1(c), we observe that this mode is associated with a strong dipolar charge oscillation within the attached remnant junction and the lower subnanorod, inherited from the weak prebreakage feature at $1.6 \mathrm{eV}$ discussed above.

Having characterized the plasmon modes in the system, we proceed to analyze in detail their evolution. Toward this aim, we show in Figs. 2(a) and 2(b) the extracted energies and intensities of the plasmon modes as a function of the elongation [52]. These figures reveal the rich structure in the evolution of the modes. The stepped evolution of the different plasmon modes is particularly remarkable. This is especially pronounced for the CTP mode, which undergoes strong intensity drops during stretching at many elongation values. We also observe discontinuous shifts in the plasmon energies at the same elongations, most notably at $d=7.5 \AA$, where the BDP and CTP modes redshift by $0.2 \mathrm{eV}$ and $0.1 \mathrm{eV}$, respectively. The discontinuities can be traced back to the rearrangements in the junction, but such strong intensity changes can hardly be explained by the geometrical change alone. Indeed, the discontinuous evolution of the intensity of the CTP mode is very similar to the quantum features shown by the conductance curves measured during the breaking of metallic nanowires [53]. 


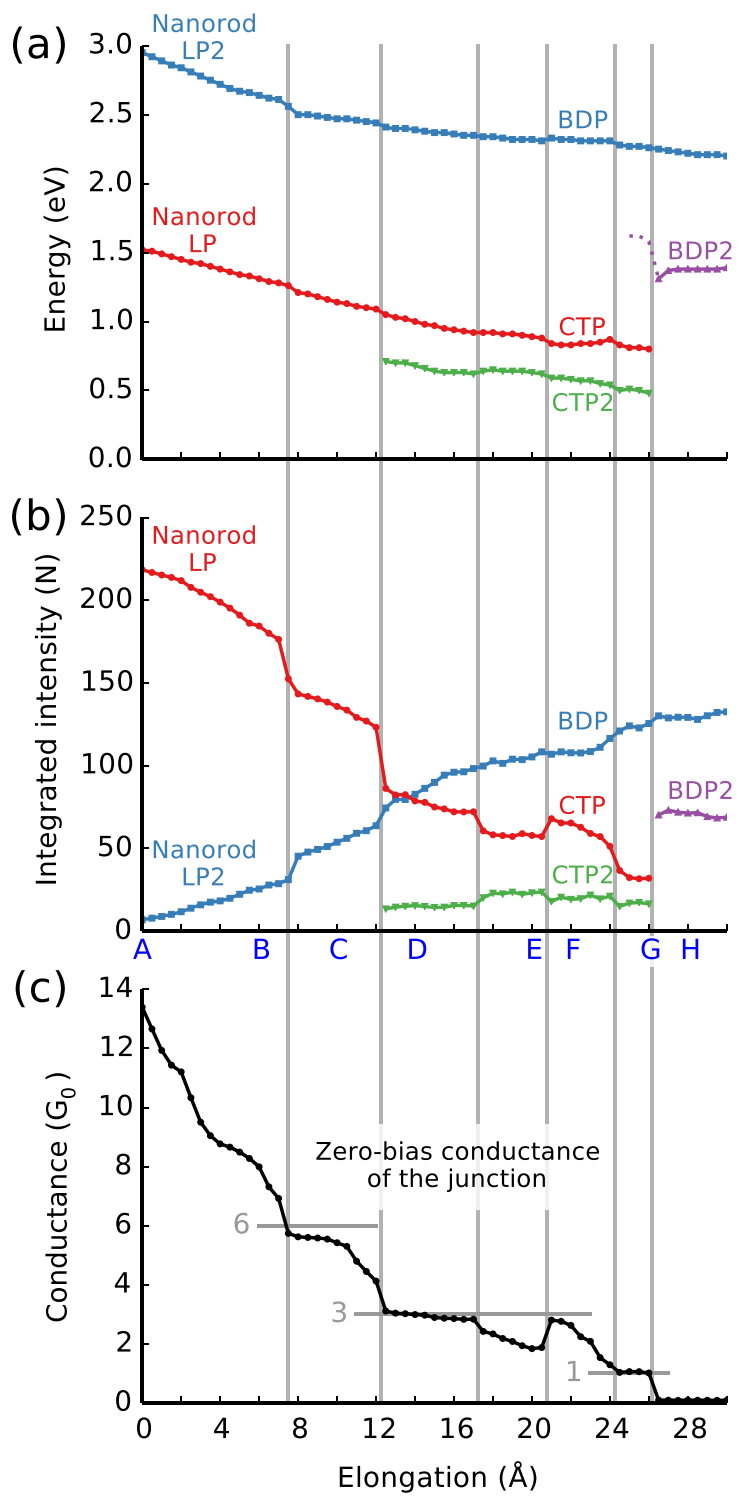

FIG. 2 (color online). Detailed analysis of the plasmon modes during the nanorod stretching. (a) Peak energy and (b) integrated intensity (the area under the peak) of the plasmon modes as a function of the nanorod elongation $d$ [52]. The intensities are normalized so that the full spectrum integrates to the number of valence electrons (261). The line labels correspond to the labeling used in Fig. 1(b) and labels $A-H$ to the geometries shown in Fig. 1(a). (c) Zero-bias conductance of the junction formed during the stretching. Horizontal lines mark values of $1 G_{0}, 3 G_{0}$, and $6 G_{0}$. Vertical lines mark the discontinuities found in the evolution of the plasmon modes.

To examine the connection between the observed discontinuities and the electron transport properties of the junction, we have calculated the zero-bias conductance of the junction by the nonequilibrium Green's function technique [54] as implemented in GPAW [55,56]. The conductance results plotted in Fig. 2(c) show the wellknown features that arise in the breaking of atomic-sized junctions from the quantization of the conduction channels
[53]: Upon stretching, in addition to the gradual evolution of conductance (reflecting changes in the transmission probabilities of conduction channels), clear jumps of integer multiples of the quantum conductance unit $G_{0}=$ $2 e^{2} / h$ take place (due to the closing of channels). When these steps are compared with the discontinuities found in the evolution of the plasmon modes, we observe a nearly one-to-one match. Thus, we conclude that upon the stretching of the nanorod, the number of conduction channels supported by the junction is reduced in a discrete manner [27] after the atomic rearrangements in the junction; this leads to abrupt changes in the allowed charge flow between the subnanorods as shown by the conductance, which is further reflected in the plasmonic response. This phase-coherent transport effect is particularly pronounced in the CTP absorption intensity, as expected from the character of the mode.

While the intensity of the CTP mode closely follows the trends shown by the conductance of the junction, even reflecting the increase of the conductance at $d \approx 21 \AA$ (ascribed to enhanced transmission due to the formation of the well-ordered junction geometry), the CTP2 mode behaves differently. This is attributed to the previously described differences in the spatial characteristics of the two modes: The reduction of the effective cross section of the junction along with the elongation uncovers the inner surfaces of the subnanorods necessary to support the CTP2 mode, and leads to its growth. The BDP mode, which also has a strong localization in the inner ends of the subnanorods, is similarly affected. Further fine details in the evolution of the spectra can be attributed to relatively complex stochastic atomic configurations.

In conclusion, we performed a first-principles study of the plasmonic response of a stretched metallic nanorod. We found a quantized evolution of the plasmon modes and established a connection between the plasmonic discontinuities and the discrete number of conduction channels supported by the break junction. Thus, this phenomenon can be considered the plasmonic counterpart of the quantization of the conductance in atomic-sized junctions. The effect is most prominent in the main charge-transfer plasmon of the considered sodium nanorod. In noble metal systems, the damping due to the $d$ electrons is weak for such a low-energy plasmon mode [58,59]; thus, we expect similar quantized evolution to be observable in these materials also. As our findings are based on the formation of a thin nanocontact, they are most likely to be experimentally reproducible in a contact-breaking situation $[51,60]$ of nanoparticle dimers rather than in a jump to contact $[9,10,16,17,48]$.

We thank the Academy of Finland for support through its Centres of Excellence Programme (2012-2017) under Projects No. 251748 and No. 284621. T. P. R. acknowledges financial support from the Vilho, Yrjö, and Kalle Väisälä Foundation of the Finnish Academy of Science and Letters. 
We acknowledge computational resources provided by the Aalto Science-IT project and CSC-IT Center for Science Ltd. (Espoo, Finland).

Note added.-A preprint of a related study has recently appeared [61].

*tuomas.rossi@alumni.aalto.fi

[1] J. N. Anker, W. P. Hall, O. Lyandres, N. C. Shah, J. Zhao, and R. P. Van Duyne, Nat. Mater. 7, 442 (2008).

[2] J. Aizpurua, G. W. Bryant, L. J. Richter, F. J. García de Abajo, B. K. Kelley, and T. Mallouk, Phys. Rev. B 71, 235420 (2005).

[3] X. Huang, S. Neretina, and M. A. El-Sayed, Adv. Mater. 21, 4880 (2009).

[4] L. O. Herrmann, V. K. Valev, C. Tserkezis, J. S. Barnard, S. Kasera, O. A. Scherman, J. Aizpurua, and J. J. Baumberg, Nat. Commun. 5, 4568 (2014).

[5] L. Novotny and N. van Hulst, Nat. Photonics 5, 83 (2011).

[6] G. Georgiou, C. Tserkezis, M. C. Schaafsma, J. Aizpurua, and J. Gómez Rivas, Phys. Rev. B 91, 125443 (2015).

[7] E. Prodan, C. Radloff, N. J. Halas, and P. Nordlander, Science 302, 419 (2003).

[8] P. Nordlander, C. Oubre, E. Prodan, K. Li, and M. I. Stockman, Nano Lett. 4, 899 (2004).

[9] K. J. Savage, M. M. Hawkeye, R. Esteban, A. G. Borisov, J. Aizpurua, and J. J. Baumberg, Nature (London) 491, 574 (2012).

[10] J. A. Scholl, A. García-Etxarri, A. L. Koh, and J. A. Dionne, Nano Lett. 13, 564 (2013).

[11] J. Zuloaga, E. Prodan, and P. Nordlander, Nano Lett. 9, 887 (2009).

[12] R. Esteban, A. G. Borisov, P. Nordlander, and J. Aizpurua, Nat. Commun. 3, 825 (2012).

[13] D. Marinica, A. Kazansky, P. Nordlander, J. Aizpurua, and A. G. Borisov, Nano Lett. 12, 1333 (2012).

[14] W. Yan, M. Wubs, and N. Asger Mortensen, Phys. Rev. Lett. 115, 137403 (2015).

[15] P. Zhang, J. Feist, A. Rubio, P. García-González, and F. J. García-Vidal, Phys. Rev. B 90, 161407 (2014).

[16] A. Varas, P. García-González, F. J. García-Vidal, and A. Rubio, J. Phys. Chem. Lett. 6, 1891 (2015).

[17] M. Barbry, P. Koval, F. Marchesin, R. Esteban, A. G. Borisov, J. Aizpurua, and D. Sánchez-Portal, Nano Lett. 15, 3410 (2015).

[18] P. Song, P. Nordlander, and S. Gao, J. Chem. Phys. 134, 074701 (2011).

[19] P. Song, S. Meng, P. Nordlander, and S. Gao, Phys. Rev. B 86, 121410 (2012).

[20] S. F. Tan, L. Wu, J. K. Yang, P. Bai, M. Bosman, and C. A. Nijhuis, Science 343, 1496 (2014).

[21] V. Kulkarni and A. Manjavacas, ACS Photonics 2, 987 (2015).

[22] R. Takahata, S. Yamazoe, K. Koyasu, and T. Tsukuda, J. Am. Chem. Soc. 136, 8489 (2014).

[23] R. N. Barnett and U. Landman, Nature (London) 387, 788 (1997).

[24] A. Nakamura, M. Brandbyge, L. B. Hansen, and K. W. Jacobsen, Phys. Rev. Lett. 82, 1538 (1999).
[25] E. Ogando, T. Torsti, N. Zabala, and M. J. Puska, Phys. Rev. B 67, 075417 (2003).

[26] M. Koskinen, P. Lipas, and M. Manninen, Z. Phys. D 35, 285 (1995).

[27] See Supplemental Material at http://link.aps.org/ supplemental/10.1103/PhysRevLett.115.236804, which includes Refs. [28-32], for discussion on the smoothing of the IID maps and analysis of the junction electronic structure and transport properties.

[28] S. Gao and Z. Yuan, Phys. Rev. B 72, 121406 (2005).

[29] S. Bernadotte, F. Evers, and C. R. Jacob, J. Phys. Chem. C 117, 1863 (2013).

[30] J. Zuloaga, E. Prodan, and P. Nordlander, ACS Nano 4, 5269 (2010).

[31] P. E. Blöchl, Phys. Rev. B 50, 17953 (1994).

[32] D. K. Ferry, S. M. Goodnick, and J. Bird, Transport in Nanostructures, 2nd ed. (Cambridge University Press, Cambridge, England, 2009).

[33] P. Hohenberg and W. Kohn, Phys. Rev. 136, B864 (1964); W. Kohn and L. J. Sham, Phys. Rev. 140, A1133 (1965).

[34] J. Enkovaara et al., J. Phys. Condens. Matter 22, 253202 (2010); A. H. Larsen, M. Vanin, J. J. Mortensen, K. S. Thygesen, and K. W. Jacobsen, Phys. Rev. B 80, 195112 (2009); J. J. Mortensen, L. B. Hansen, and K. W. Jacobsen, Phys. Rev. B 71, 035109 (2005).

[35] The atom positions were relaxed by a quasi-Newton method. Atomic forces were converged to less than $0.01 \mathrm{eV} / \AA$. Calculations were done within the linear combination of atomic orbitals (LCAO) description using the double- $\zeta$ polarized basis set, grid spacing of $0.4 \AA$, and vacuum space around the system $\geq 6 \AA$.

[36] J. P. Perdew, K. Burke, and M. Ernzerhof, Phys. Rev. Lett. 77, 3865 (1996); 78, 1396 (1997).

[37] A. I. Yanson, I. K. Yanson, and J. M. van Ruitenbeek, Nature (London) 400, 144 (1999).

[38] P. Jelínek, R. Pérez, J. Ortega, and F. Flores, Phys. Rev. B 77, 115447 (2008).

[39] A. Zugarramurdi, A. G. Borisov, N. Zabala, E. V. Chulkov, and M. J. Puska, Phys. Rev. B 83, 035402 (2011).

[40] S. Barzilai, F. Tavazza, and L. E. Levine, J. Phys. Condens. Matter 25, 325303 (2013).

[41] E. Runge and E. K. U. Gross, Phys. Rev. Lett. 52, 997 (1984).

[42] K. Yabana and G. F. Bertsch, Phys. Rev. B 54, 4484 (1996).

[43] M. Walter, H. Häkkinen, L. Lehtovaara, M. Puska, J. Enkovaara, C. Rostgaard, and J. J. Mortensen, J. Chem. Phys. 128, 244101 (2008).

[44] M. Kuisma, A. Sakko, T. P. Rossi, A. H. Larsen, J. Enkovaara, L. Lehtovaara, and T. T. Rantala, Phys. Rev. B 91, 115431 (2015).

[45] TDDFT calculations were performed on a real-space grid with grid spacing of $0.4 \AA$ and zero boundary conditions. The size of the calculation cell was set to $25.6 \times 25.6 \times$ $96.0 \AA^{3}$, ensuring a vacuum space around each geometry $\geq 6 \AA$. The Hartree potential was evaluated in a larger cell of $64 \times 64 \times 128 \AA^{3}$ (vacuum space $\geq 24 \AA$ ). The propagation time step was 20 as. A Gaussian folding with a full width at half maximum of $0.19 \mathrm{eV}$ was applied to the spectra.

[46] L. Liu, Y. Wang, Z. Fang, and K. Zhao, J. Chem. Phys. 139, 064310 (2013). 
[47] O. Pérez-González, N. Zabala, and J. Aizpurua, New J. Phys. 13, 083013 (2011).

[48] H. Duan, A. I. Fernández-Domínguez, M. Bosman, S. A. Maier, and J. K. W. Yang, Nano Lett. 12, 1683 (2012).

[49] I. Alber, W. Sigle, F. Demming-Janssen, R. Neumann, C. Trautmann, P. A. van Aken, and M. E. Toimil-Molares, ACS Nano 6, 9711 (2012).

[50] J. Fontana and B. R. Ratna, Appl. Phys. Lett. 105, 011107 (2014).

[51] F. Wen, Y. Zhang, S. Gottheim, N. S. King, Y. Zhang, P. Nordlander, and N. J. Halas, ACS Nano 9, 6428 (2015).

[52] We fitted Voigt profiles to the polarizability spectrum $\operatorname{Im}[\alpha(\omega)]$ and extracted the peak parameters of the absorption spectrum via Eq. (1). We checked that the obtained trends are robust with respect to the fitting procedure.

[53] N. Agraït, A. L. Yeyati, and J. M. van Ruitenbeek, Phys. Rep. 377, 81 (2003).

[54] J. Taylor, H. Guo, and J. Wang, Phys. Rev. B 63, 245407 (2001).

[55] J. Chen, K. S. Thygesen, and K. W. Jacobsen, Phys. Rev. B 85, 155140 (2012).
[56] The conductance calculations require us to sandwich the junction between two periodic semi-infinite leads. This was accomplished by infinitely extending the frozen ends of the nanorod. In these calculations we used a single- $\zeta$ basis set augmented with one $s$ - and two $p$-type truncated Gaussiantype orbitals that were generated for optimally describing the photoabsorption of sodium nanoparticles by using the methodology in Ref. [57].

[57] T. P. Rossi, S. Lehtola, A. Sakko, M. J. Puska, and R. M. Nieminen, J. Chem. Phys. 142, 094114 (2015).

[58] C. Sönnichsen, T. Franzl, T. Wilk, G. von Plessen, J. Feldmann, O. Wilson, and P. Mulvaney, Phys. Rev. Lett. 88, 077402 (2002).

[59] X. López-Lozano, H. Barron, C. Mottet, and H.-C. Weissker, Phys. Chem. Chem. Phys. 16, 1820 (2014).

[60] M.-M. Mennemanteuil, J. Dellinger, M. Buret, G. Colas des Francs, and A. Bouhelier, Appl. Phys. Lett. 105, 021101 (2014).

[61] F. Marchesin, P. Koval, M. Barbry, J. Aizpurua, and D. Sánchez-Portal, arXiv:1509.07194v1. 